\title{
Developing Machine Learning Models for Ionic Conductivity of Imidazolium-Based Ionic Liquids
}

\author{
Pratik Dhakal and Jindal K. Shah ${ }^{1}$ \\ School of Chemical Engineering, Oklahoma State University, Stillwater, Oklahoma,74078, \\ United States
}

\begin{abstract}
In this study, we developed two machine learning models, support vector machine (SVM) and artificial neural network (ANN), to correlate ionic conductivity of pure ionic liquids based on the imidazolium cations using the data acquired from the NIST ILThermo database. Both models were shown to successfully capture the entire range of ionic conductivity spanning six orders of magnitude over a temperature range of $275-475 \mathrm{~K}$ with relatively low statistical uncertainty. Due to slightly better performance, ANN was used to predict the ionic conductivity for 1102 ionic liquids formed from every possible combination of 29 cations and 38 anions contained in the database. The procedure led to the generation of many ionic liquids for which the ionic conductivity was estimated to be greater than $1 \mathrm{~S} / \mathrm{m}$. The ionic liquid dimethylimidazolium dicyanamide, not present in the original dataset, was identified to exhibit the ionic conductivity of $3.70 \mathrm{~S} / \mathrm{m}$, roughly $30 \%$ higher than the highest conductivity reported for any ionic liquid at $298 \mathrm{~K}$ in the database. The ANN model was also found to accurately predict the ionic conductivity for several ionic liquid-ionic liquid mixtures, for which experimental data are available. Encouraged by this result, we calculated ionic conductivity for all the possible binary ionic liquid-ionic liquid mixtures based on the cations and anions contained in ?he dataset. The model predictions revealed a large number of ionic liquid mixtures systems exhibiting nonideal behavior where a maximum or minimum in the ionic conductivity was observed as a function of composition, similar to trends seen in binary ionic liquid mixture of water or conventional solvents with ionic liquids.
\end{abstract}

Keywords: Ionic liquids; ionic liquid-ionic liquid mixtures; ionic conductivity; machine learning; artificial neural network; support vector machine

* Corresponding author 


\section{Introduction}

Room temperature ionic liquids are a class of salts that are liquid at room temperature consisting exclusively of ions. They are currently one of the most studied solvents because of several unique properties such as negligible volatility, electrochemical stability, low melting point, and high thermal and chemical stability [1. Because of all of these desirable properties, ionic liquids are investigated for various industrial applications such as potential solvents to break minimum/maximum boiling azeotropes 2, 3, 4, 5, extracting agent in LLE separations 6, 7, 8, 9, electrolytes in electrochemical devices [10, 11, 12, 13, and solvent for gas-capture [14, 15, 16, 17. Despite the various favorable attributes inherent in an ionic liquid, high viscosity and low ionic conductivity of many ionic liquids, especially at low temperatures, is a bottleneck for the application of ionic liquids as electrolytes in batteries. [18]

A widely adopted approach to mitigate potential drawbacks for using ionic liquids is to tune the properties of an ionic liquid by altering functional group(s) attached to the cation, changing the cationic core (e.g. from aromatic to cyclic), and/or modifying the chemical composition of the anion. Developing new ionic liquids this way requires considerable chemical intuition, expertise in synthesis, and subsequent measurements of properties. Given the breadth of the chemical space for cations and anions, it is practically impossible to study every possible combination of the cation and anion. The explosion in the chemical space is further exacerbated by the increasing popularity of exploiting ionic liquid-ionic liquid mixtures for tailoring properties of these solvents. [19, 20, 21] One estimate projects that there are as many as one billion ionic liquid systems. [22]. Although daunting from an experimental or molecular simulation viewpoint, the vast chemical space of cations and anions also offers a unique opportunity to leverage machine learning and data analytics-based techniques to search and design ionic liquids with properties suited for a given application.

Indeed, several studies over the years have used artificial neural network ANN to model and predict ionic liquid properties such as density, 23 viscosity 24,25 , melting point [26, toxicity [27, solubility of gases, such as $\mathrm{CO}_{2}$ [28, 29] and $\mathrm{SO}_{2}$ [30] in ionic liquids, surface tension [31, investigating ionic liquid-solvent mixtures, 32, 33, 34, 35, and prediction of rate constants in ionic liquid-organic mixtures [36]. Additional examples involving the application of ANN for various properties for ionic liquids can be found in a recent review article by Yusuf et al. 37] ARecently, Beckner and Pfaendtner have demonstrated that it is possible to combine machine learning and genetic algorithm to develop new ionic liquids with high thermal conductivity. 38] Some advances have also occurred for correlating ionic conductivity, an extremely useful property for selecting electrolytes in electrochemical applications and the topic of the present article. Krossing et al. used the concept of free volume and derived an empirical equation based on Cohen-Turnbull free volume theory to correlate transport properties such as ionic conductivity and viscosity for imidazolium based ionic liquids that were 
in good agreement with experimental data at high temperature range, while some deviations were noted in the low temperature regime. 39. Passerini et al. found that the molar conductivity of pyrrolidinium based cations paired with sulfonylimide anions showed a high correlation $\left(R^{2}=0.9942\right)$ with the sum of cation and anion volumes obtained from electronic structure calculations. 40] The observation suggested that the molar ionic conductivity decreased with an increase in the combined volume. However, no such monotonicity existed for imidazolium-based ionic liquids, which is the focus of the present study. Beichel et al. used volume-based thermodynamics (VBT) approach to correlate ionic conductivity of ionic liquids based on parameters such as molecular volume and surface area calculated using COnductor-like Screening MOdel(COSMO). [41] The authors reported an overall root mean square error of 0.04-0.06 $\log (\sigma)$. Group contribution (GC) methods have also been found useful for developing a correlation between the ionic conductivity and various chemical features of ionic liquids. For example, Gharagheizi et al. employed a least-squared support vector machine GC method to estimate ionic conductivity consisting of a dataset with 54 different unique ionic liquids with an absolute average relative deviation (AARD) of 3.3\% [42]. Tochigi et al. developed a polynomial-based quantitative structure-property relationship (QSPR) to predict ionic conductivity for eight different cation families and sixteen different anions 43 . The authors reported an overall $R^{2}$ of 0.91 and standard deviation of $0.12 \mathrm{~S} / \mathrm{m}$ for 139 data points. Coutinho et al. used a three-parameter GC method equation similar to VogelTammann-Fulcher (VTF) for the estimation of ionic conductivity for pure ionic liquids. 44. Wooley et al. applied a four-parameter GC-based approach to estimate ionic conductivity of ionic liquids. 45. An attractive feature of GC methods is that chemically intuitive groups are usually selected as inputs to the model prior to optimizing model parameters. However, for billions of ionic liquids with vastly different chemical functionalities, identifying and enumerating all the relevant groups can pose significant difficulties to eventual automated screening of ionic liquids.

In this article, we explore a different approach rooted in the framework of machine learning techniques such as artificial neural network and support vector machine to correlate the ionic conductivity of pure ionic liquids. We assess the performance of the two models and examine if the model can be extended to predict ionic conductivity of all possible combinations of unique cations and anions in the database and binary ionic liquid systems. As such the next section provides details on the data collection and processing, model formulation, and model validation. In the subsequent section, the models, trained with the ionic conductivity of pure ionic liquids, are compared. The model with better accuracy is identified and is extended to predict the ionic conductivity for in silico ionic liquids obtained by enumerating possible combinations of cations and anions contained in the dataset. We will demonstrate that such a procedure leads to the discovery of the ionic liquid with the highest conductivity, which matches with the experimental data at $298 \mathrm{~K}$. The predictive capability of the model will be discussed in terms of the level of agreement for ionic conductivity for several 
binary ionic liquid systems. The possibility of obtaining enhancement in the ionic conductivity by formulating binary ionic liquid mixtures will be presented followed by a summary of findings and the direction for future research.

\section{Methodology}

\subsection{Data Collection and Processing}

A total of 2895 ionic conductivity data for pure component imidazoliumbased ionic liquid were downloaded from the online ILThermo database maintained by NIST [46, 47] using the pyILT2 [48, utility. Majority of the ionic conductivity data were measured using alternating current cell with electrodes [49, 50, while a small number of data were acquired using direct current cell with electrodes [51, 52, while a small number of articles reported the ionic conductivity obtained with capillary cell, electrochemical (EC) cell [53], impedancemetry [54, 55] and conductivimeter [56]. The downloaded data were processed (see below) and formatted with an in-house Python script. The datapoints contained the ionic liquid name, temperature $(\mathrm{K})$, pressure $(\mathrm{kPa})$, reference from which the data was extracted, and the uncertainty in the measurement. Approximately $89 \%$ of the data represented ionic conductivity in the liquid state, while $\sim 10 \%$ of the data for crystals, and a small fraction of the data with ionic conductivities for metastable liquids were discarded from the training set.

The next step involved a careful examination of the dataset. First, we eliminated any entries with missing values for the ionic conductivity or "NaN" in the dataset. To accomplish the removal of inconsistent data or typographical errors, we graphed ionic conductivity data as a function of temperature to identify outliers in the dataset. Some of the ionic conductivities were extremely low, in the range of $10^{-9} \mathrm{~S} / \mathrm{m}$ belonging to ionic liquids comprised of natural amino acids as the anions combined with 1-ethyl-3-methylimidazolium $\left[\mathrm{C}_{2} \mathrm{mim}\right]^{+}$cation at 298.0 K. [57] We eliminated these points due to very low values of ionic conductivity and the fact that the model derived from an artificial neural network (ANN, see below) could not be extended to such small values. We also found that ionic conductivities for the pure 1-n-hexyl-3-methylimidazolium $\left[\mathrm{C}_{6} \mathrm{mim}\right]$ bromide $\mathrm{Br}$ and 1-n-octyl-3-methylimidazolium $\left[\mathrm{C}_{8} \mathrm{mim}\right] \mathrm{Br}$ were reported to be $144.1 \mathrm{~S} / \mathrm{m}$ and $116.4 \mathrm{~S} / \mathrm{m}$, respectively at $333.15 \mathrm{~K}$. 58. These values are two orders of magnitude larger than those for many imidazolium-based ionic liquids. For example, ionic liquids with shorter alkyl chain length such as $\left[\mathrm{C}_{2} \mathrm{mim}\right] \mathrm{Br}$ and 1-n-butyl-3-methylimidazolium $\left[\mathrm{C}_{4} \mathrm{mim}\right] \mathrm{Br}$ have been reported to possess ionic conductivities of $1.06 \mathrm{~S} / \mathrm{m}$ at $335.6 \mathrm{~K} \mathrm{[50]}$ and $0.734 \mathrm{~S} / \mathrm{m}$ at $373.1 \mathrm{~K}$. [59] The visualization of the ionic conductivity as a function of the alkyl chain length also showed that the ionic conductivity decreases with the increase in the alkyl chain. Thus, the inconsistency led us to remove the seemingly high ionic conductivity datapoints. We pruned the dataset further by identifying duplicate ionic liquid fields (same cation, anion, temperature, and pressure) and keeping only the entry with the lowest uncertainty in the ionic conductivity measurements. 
We further reduced the number of points for model development by visualizing the data to obtain a clue into the appropriate ranges for the ionic conductivity, temperature and pressure along with chemical identities of the ionic liquid in the database. We observed that a large fraction of the measurements have been conducted in the temperature range spanning 275-475 K (Figure S1). Thus, we removed all the data points outside this temperature range. As there were only a limited number of points present at pressures other than $101 \mathrm{kPa}$, we decided to restrict the model development by fixing the pressure at $101 \mathrm{kPa}$. The resulting dataset contained a total of 1323 data points with ionic conductivities over six orders of magnitude from $4.1 \times 10^{-5} \mathrm{~S} / \mathrm{m}$ to $19.3 \mathrm{~S} / \mathrm{m}$ as seen in supporting information. To assess the variability in the chemical identities of the cations and anions represented in the data set, we generated Figure 1 for every ionic liquid for which more than five data points were present; the size of the marker in the figure is proportional to the number of points reported for each of the ionic liquids. It is clear that a large fraction of the ionic conductivity measurements cover the cations $\left[\mathrm{C}_{2} \mathrm{mim}\right]^{+}$and $\left[\mathrm{C}_{4} \mathrm{mim}\right]^{+}$paired with a broad variety of anions, while the remaining cations, on an average, are combined with two to three distinct anions. Overall, we found that the dataset contained 29 unique cations and 38 unique anions. There were a total of 111 ionic liquids, approximately $10 \%$ of the ionic liquids that could be formed by combining cations and anions from the dataset.

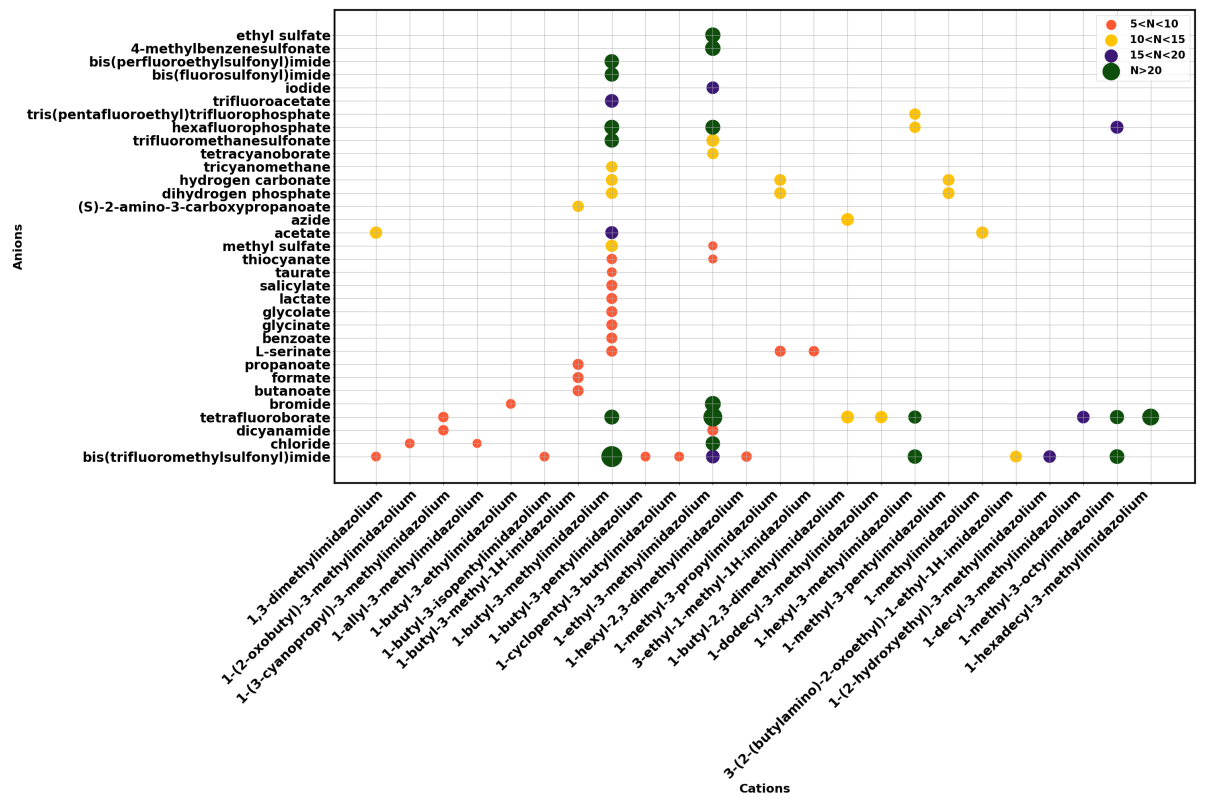

Figure 1: The number of ionic liquids for a given cation-anion combination with more than five data points in the NIST ILThermo database is shown. 


\subsection{Feature Generation and Elimination}

We translated the identities of the cations and anions to simplified molecularinput line-entry system or SMILES format using an open-source online website Open Parser for Systematic IUPAC Nomenclature OPSIN. 60, 61] One of the anions in the dataset [tetrakis(isothiocyanto)cobaltate $\left(2^{-}\right)$] could not be converted to SMILES format, therefore we removed the anion and corresponding ionic liquids from further consideration. We used an open-source cheminformatics package RDKit 62 to generate descriptor features for the input to the ANN. RDKit produced a total of 196 descriptors for each of the cations and anions. A complete listing is available in the supporting information. Prior to utilizing these features in the model development, we examined the correlation among features to reduce the dimensionality of the input and increase the speed of learning algorithms by calculating cross correlation coefficients for every feature with every other feature. Comparing the correlation coefficients sequentially, we eliminated any feature that showed either a positive or negative correlation coefficient of greater than 0.9 with any of the previous features. This process brought the aggregate number of cation and anion features down to 38 and 59, respectively, for a total of 99 chemical features including temperature and pressure for a given ionic liquid. The final set of features used below for the model development is included in the supporting information.

\subsection{Data Normalization}

Data normalization is a standard technique in improving the model performance and minimizing biases in a multivariate regression with feature values varying over a wide range. For instance, the RDKit feature 'hydrogen count' would possess a considerably smaller range of values for the cations and anions in comparison to those for the 'molecular weight' feature, which will likely influence the weightage assigned to these features. On the output side, the ionic conductivity data varied over six orders of magnitude as pointed out earlier. Therefore, we decided to use MinMaxScaler implemented in Scikit-learn 63] to normalize each input feature and the output by the difference in the maximum and minimum values, which led to any feature or output value to fall between 0 and 1 . We preserved the scaling employed during the model generation for later use in the prediction.

\subsection{Model Development}

In this work, we used a total of 1323 experimental data points with a focus on cations exclusively from the imidazolium family to build machine learning model. The training set consisted of $90 \%$ of the total data, while the remaining $10 \%$ of the data was used as test case to evaluate the model's performance. The model was constructed using two of the most popular machine learning methods, support vector machine for regression (SVR) and feed-forward artificial neural network (FFANN). 
Support vector machine (SVM) is a supervised machine learning framework used for classification and regression problems. 64, 65, 66] The regression version of SVM is called support vector regression (SVR) with the central objective of finding the best fit line in the hyperplane that touches a maximum number of points. Hyper-parameter tuning of SVR parameters is extremely important to improve the model's accuracy for regression analysis. Similarly, feed-forward artificial neural network (FFANN) is also a supervised learning technique with a mapping function $y=f(\mathbf{x} ; \theta)$ where $\theta$ is the parameter set that the model learns to provide the most optimal approximation of the function based on the input feature vector $\mathbf{x}$. The FFANN consisted of three layers: an input layer, a hidden layer and the output layer. The input layer consisted of chemical features along with the state points temperature $\mathrm{T}$ and pressure $\mathrm{P}$.

Hyper parameters for both the models were tuned using GridSearchCV implemented in Scikit-learn 63. GridSearchCV exhaustively searches all the hyper-parameter combination listed in the parameter search space to identify the best performing hyper-parameters. The search space for both the models along with the final hyper-parameters are provided in the supporting information. The GridSearchCV method is combined with $10 \mathrm{~K}$-Fold cross validation to avoid any overfitting during the hyper-parameter search. The best performing model architecture with the highest accuracy during this hyper-parameter tuning process was selected as the final model with a further evaluation conducted on the test case set aside earlier. The workflow for cross-validation and testing of the model is depicted in Figure 2 .

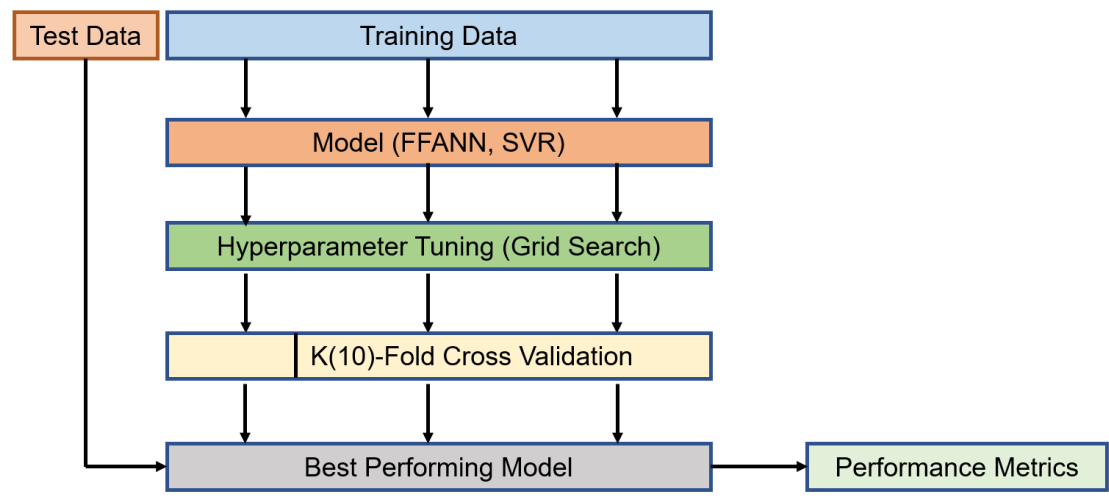

Figure 2: Description of model development followed in this study.

\section{Results and Discussion}

\subsection{Model Validity}

In this work, we developed machine learning model to predict ionic conductivity of imidazolium ionic liquids using two different techniques. The best- 
performing model produced the lowest statistical uncertainty and captured the trends in the data such as the lowest ten and the top ten ionic conductivity measurements. During the model development, we observed that the models based on MinMax scaling as discussed earlier performed extremely well for predicting conductivity values in the higher magnitude range, but the predictive capability greatly diminished in the lower conductivity region. For instance, the experimental value of $\left[\mathrm{C}_{6} \mathrm{mim}\right]$ tetrafluoroborate $\left[\mathrm{BF}_{4}\right]$ is $6.7 \times 10^{-4} \mathrm{~S} / \mathrm{m}$ at $298 \mathrm{~K}$; however, the predicted value was $3.3 \times 10^{-2} \mathrm{~S} / \mathrm{m}$ - an error of two orders of magnitude. We noted this behavior for many other ionic liquid systems with ionic conductivity values on the lower end. The observation prompted us to convert the ionic conductivity values on a logarithm scale (base 10) before applying the MinMax scaling, which led to a dramatic improvement in the prediction of low ionic conductivity values. For example, the ionic conductivity prediction for the ionic liquid $\left[\mathrm{C}_{6} \mathrm{mim}\right]\left[\mathrm{BF}_{4}\right]$ was $7.2 \times 10^{-4} \mathrm{~S} / \mathrm{m}$ in comparison to experimental measurement of $6.7 \times 10^{-4} \mathrm{~S} / \mathrm{m}$.

Table 1: Comparison of the predictions results for FFANN and SVR for the training set, test set and the entire dataset. MSE is the mean squared error, MAE is the mean absolute error, RMSD is root mean square deviation and $R^{2}$ is the squared correlation between experiment and predicted data. $\log _{10}$ scale refers to ionic conductivity scaled to $\log _{10}$, while normal scale refers to ionic conductivity data without any scaling in $\mathrm{S} / \mathrm{m}$.

\begin{tabular}{cccccccc}
\hline Scale & Metric & \multicolumn{2}{c}{ Train } & \multicolumn{2}{c}{ Test } & \multicolumn{2}{c}{ Entire } \\
\hline $\log _{10}$ scale & & SVR & FFANN & SVR & FFANN & SVR & FFANN \\
& $R^{2}$ & 0.995 & 0.993 & 0.976 & 0.99 & 0.993 & 0.994 \\
& MSE & 0.002 & 0.003 & 0.012 & 0.004 & 0.0032 & 0.003 \\
& MAE & 0.014 & 0.036 & 0.038 & 0.044 & 0.017 & 0.037 \\
& RMSD & 0.047 & 0.057 & 0.111 & 0.071 & 0.057 & 0.059 \\
\hline Normal scale & $R^{2}$ & 0.999 & 0.995 & 0.997 & 0.996 & 0.999 & 0.996 \\
& MSE & 0.003 & 0.021 & 0.023 & 0.024 & 0.005 & 0.022 \\
& MAE & 0.027 & 0.079 & 0.059 & 0.086 & 0.031 & 0.081 \\
& RMSD & 0.061 & 0.148 & 0.153 & 0.016 & 0.075 & 0.149 \\
\hline
\end{tabular}

Table 1 details the statistical assessment of the two models for the training set, test dataset and the entire set. The performance metrics is further divided into $\log _{10}$ scale on which the model was trained and the normal ionic conductivity scale to examine the deviation between experiment and predicted data. Both the models not only perform well for the training set, but they also have very high $R^{2}$ and low MSE, MAE and RMSD for the test set. Surprisingly, the performance metrics for the normal scale are equivalent or in some cases better than the $\log _{10}$ scale despite the model not being trained on the normal scale. 

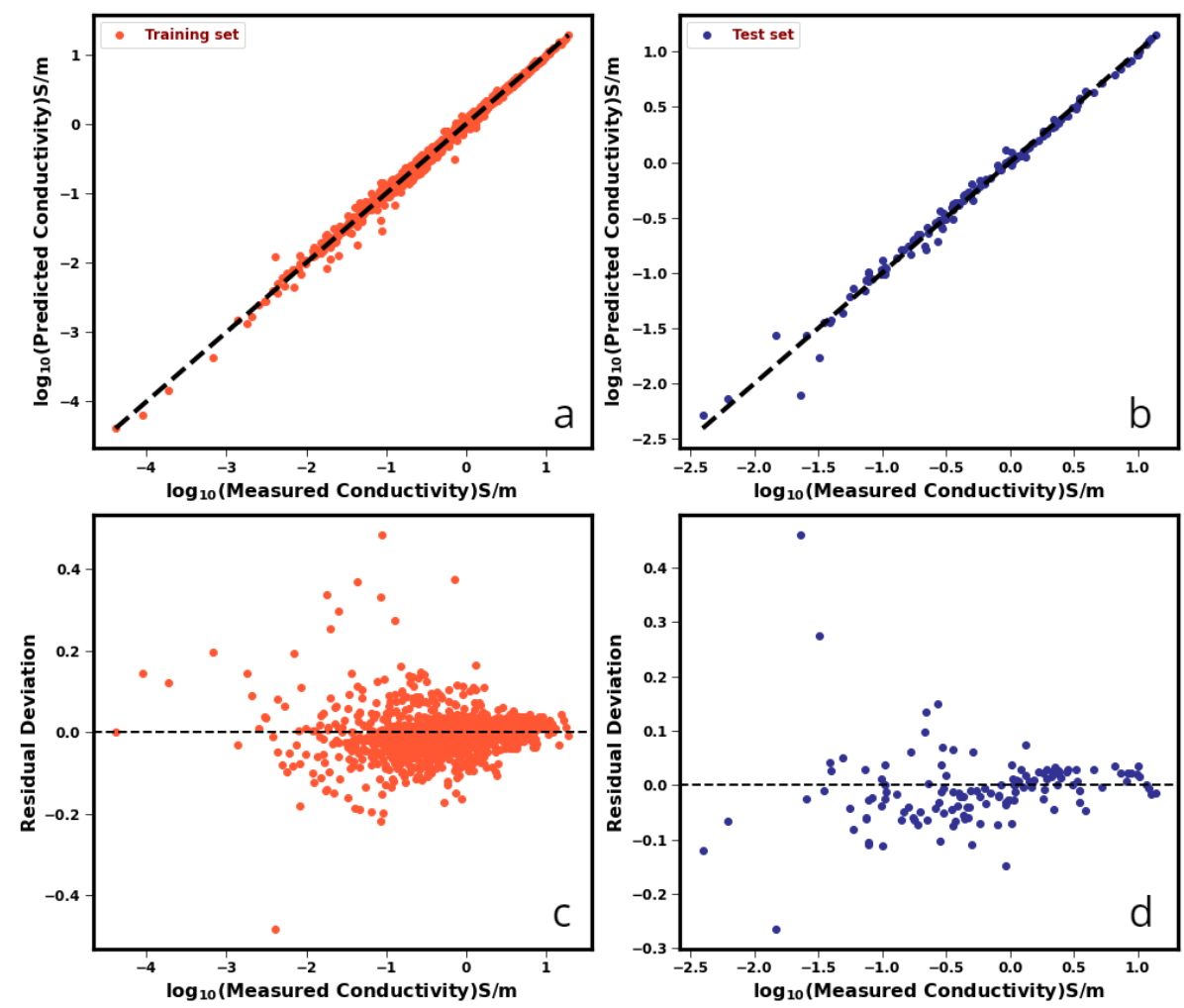

Figure 3: (a) Comparison of FFANN model predictions with the experimental data on a $\log _{10}$ scale for the training set. A perfect prediction would fall on the $y=x$ dotted line; (b) comparison for the test set (c) Residual deviation on the log-10 scale calculated as $\left(\sigma_{\text {experiment }}-\sigma_{\text {prediction }}\right)$ where $\sigma$ refers to the ionic conductivity for the training set; (d) Residual deviation for the test set.

Figure 3(a) demonstrates that the FFANN model is able to capture the training data on the base-10 logarithmic scale spanning six orders of magnitude with a high accuracy in the low conductivity range. Figures 3 (c) and (d) show the residual deviation calculated by taking the difference in experiment and predicted value vs the experimental data. It is important to note that the residual deviation stays within $\pm 0.5 \log$ unit for the training set and the test set over the entire range of the experimental data. 

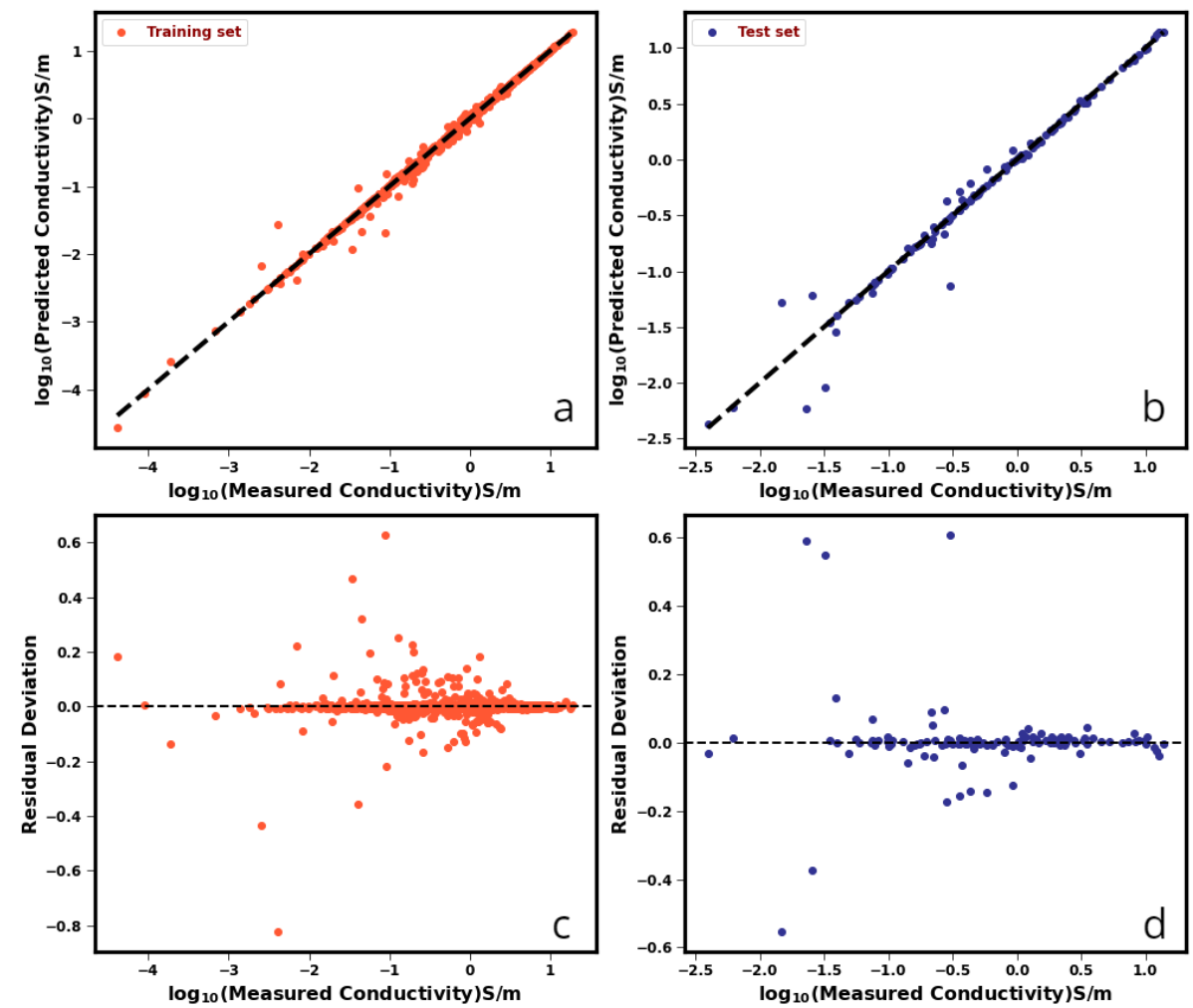

Figure 4: (a) Comparison of SVR model predictions with the experimental data on a log10 scale for the training set. A perfect prediction would fall on the $y=x$ dotted line; (b) comparison for the test set (c) Residual deviation on the log-10 scale calculated as $\left(\sigma_{\text {experiment }}-\sigma_{\text {prediction }}\right)$ where $\sigma$ refers to the ionic conductivity for the training set; (d) Residual deviation for the test set.

Similarly, Figure 4(a) depicts that the ionic conductivity correlation using the SVR model for the training set and test set. In contrast to FFANN, SVR seems to have more deviation from the $\mathrm{y}=\mathrm{x}$ line for both sets at low ionic conductivity values. This is also reflected on the residual deviation plot Figure 4(c) and (d) where the maximum deviation reaches as high as $\pm 0.6 \log$ unit for the training set. The normal scale ionic conductivity correlation using FFANN and SVR are provided in the supporting information as seen in Figure $[$ S7] and Figure $\mathrm{S} 8$.

The overall accuracy of both the model outputs is encouraging, especially when considered in the context of the ionic conductivity calculated from molecular simulations. Transport properties such as ionic conductivity are quite challenging to accurately predict from atomistic simulations requiring long simulation times and optimization of force field parameters. The problem is further exacerbated for sluggish ionic liquids possessing extremely low ionic conductivity as probed here. In such scenarios, the simulation results of ionic conductivity can 
differ by a factor up to 10 (by 1 unit on $\log _{10}$-scale) from the corresponding experimental observations. An added advantage of the proposed model is to provide guidance, at almost no computational cost, on the ionic conductivity values for in silico generated pure ionic liquids and mixtures of binary ionic liquids obtained from possible combinations of cations and anions studied here. However, we submit that the machine learning model cannot provide molecularlevel insight that is inherent in molecular simulations. For the discussion below, we focus on FFAAN as the accuracy of the model is slightly better for the entire data set.

We also probed the accuracy with which the FFAAN model captured trends. For this, we chose the data at $298 \mathrm{~K}$ as there were only a few systems for which the data was available over the entire temperature window. In Figure S9, we compare the predictions of the FFANN model for the ten lowest ionic conductivity values reported in the NIST ILThermo Database at $298 \mathrm{~K}$. We observe that the model accurately predicts the ordering of the ionic liquids while the ionic conductivities are also in very good agreement. The plot also reveals that long alkyl chains or amino acid-based anions tend to produce low conductivity ionic liquids. Similarly, Figure S10 represents a comparison between the FFANN model predictions and experimental measurements for the ten largest conductivity values at $298 \mathrm{~K}$. It is evident that the predictive capability of the model is excellent. It is also important to highlight that not only does the model capture the quantitative trend accurately, but it is also performs correctly in terms of taking into account the cation and anion properties and behavior. For example, $\left[\mathrm{BF}_{4}\right]^{-}$when paired with a long alkyl chain cation $\left[\mathrm{C}_{12} \mathrm{mim}\right]^{+}$yields one of the lowest ionic conductivity ionic liquids, while its combination with $\left[\mathrm{C}_{2} \mathrm{mim}\right]^{+}$ generates an ionic liquid with five orders of magnitude higher ionic conductivity than that for $\left[\mathrm{C}_{12} \mathrm{mim}\right]\left[\mathrm{BF}_{4}\right]$. We also point out that the change in the identity of the anion can dramatically affect the ionic conductivity as exemplified by 1allyl-3-methylimidazolium [AMIm][Benzoate] and [AMIm][Formate], the latter with the ionic conductivity four orders of magnitude higher than that for the former; the model successfully predicts the trend.

\subsection{Unique Ionic Liquid Combination}

Next we generated all the combinations of 29 unique cations and 38 anions present in the dataset, which resulted into 1102 pure ionic liquids at $298 \mathrm{~K}$ for which we predicted ionic conductivity at $298 \mathrm{~K}$. Rather than using both SVR and FFANN, we, first, tested the accuracy for such predictions using FFANN and SVR model based on two test cases that were not part of the training set. The first system is $\left[\mathrm{C}_{2} \mathrm{mim}\right]$ bis(fluorosulfonyl)imide [FSI]. The database contained $\left[\mathrm{C}_{4} \mathrm{mim}\right][\mathrm{FSI}]$ as the only ionic liquid containing $[\mathrm{FSI}]^{-}$. The model prediction for the ionic conductivity of using FFANN was found to be $1.60 \mathrm{~S} / \mathrm{m}$ for $\left[\mathrm{C}_{2} \mathrm{mim}\right][\mathrm{FSI}]$ at $298.15 \mathrm{~K}$, in excellent agreement with the corresponding experimental measurements of $1.61 \pm 0.02 \mathrm{~S} / \mathrm{m}$ compared to significantly under predicted value of $0.189 \mathrm{~S} / \mathrm{m}$ using SVR method. 67. The second system is represented by the ionic liquid $\left[\mathrm{C}_{1} \mathrm{mim}\right][\mathrm{DCA}]$, which was predicted to possess the 
highest ionic conductivity of $3.70 \mathrm{~S} / \mathrm{m}$ at $298 \mathrm{~K}$ using FFANN, which is roughly $30 \%$ higher than the highest ionic conductivity of $2.83 \mathrm{~S} / \mathrm{m}$ for $\left[\mathrm{C}_{2} \mathrm{mim}\right][\mathrm{DCA}]$. We found two experimental papers confirming that the ionic conductivity of $\left[\mathrm{C}_{1} \mathrm{mim}\right][\mathrm{DCA}]$ at $298.0 \mathrm{~K}$ is around $3.60 \mathrm{~S} / \mathrm{m}, 668,69$, once again in excellent agreement with the value obtained from our FFAN model. The SVR model, however, suggested the ionic conductivity to be $0.061 \mathrm{~S} / \mathrm{m}$, a significant underprediction. These observations point to the fact that the FFANN model is well suited to estimate ionic conductivity for ionic liquids as long as the constituent ions are present in the training set and the features for the ions generated are also present in the dataset. However that is not the case for SVR which seems to perform poorly for ionic liquids beyond the training set. It is also worth pointing out that we are able to obtain the ionic conductivity for $\left[\mathrm{C}_{1} \mathrm{mim}\right][\mathrm{DCA}]$ higher than the largest value of $2.83 \mathrm{~S} / \mathrm{m}$ at $298 \mathrm{~K}$ because the model was fitted using the ionic conductivity data up to $\sim 19 \mathrm{~S} / \mathrm{m}$ (see Figure S7(a).)

The high accuracy of the FFAAN to model the experimental ionic conductivity data prompted us to generate ionic liquid predictions as seen in Figure 5 along with the experimental ionic conductivity values at $298 \mathrm{~K}$. It is clear that a large fraction $(87.3 \%)$ of the ionic liquids exhibit ionic conductivity below $0.5 \mathrm{~S} / \mathrm{m}$. More interestingly, the procedure yielded a number of ionic liquids (approximately $8.3 \%$ ) with ionic conductivity between $0.5 \mathrm{~S} / \mathrm{m}$ and $1.0 \mathrm{~S} / \mathrm{m}$ while 47 ionic liquids were predicted to possess ionic conductivity greater than $1.0 \mathrm{~S} / \mathrm{m}$. As a comparison, the original data contained a very few ionic liquids crossing this threshold (five out of 73 data points). Cyano-based anions such as dicyanamide $\left[\mathrm{N}(\mathrm{CN})_{2}\right]^{-}$, tricyanomethanide $\left[\mathrm{C}(\mathrm{CN})_{3}\right]^{-}$, tetracyanoborate $\left[\mathrm{B}(\mathrm{CN})_{4}\right]^{-}$, and thiocyanate $[\mathrm{SCN}]^{-}$accounted for the two thirds of the ionic liquids with ionic conductivity greater than $1 \mathrm{~S} / \mathrm{m}$. As for the cation, $\left[\mathrm{C}_{1} \mathrm{mim}\right]^{+},\left[\mathrm{C}_{2} \mathrm{mim}\right]^{+}$, and $\left[\mathrm{C}_{3} \mathrm{mim}\right]^{+}$were found in two thirds of the ionic liquids for which the ionic conductivity is greater than $1 \mathrm{~S} / \mathrm{m}$. 

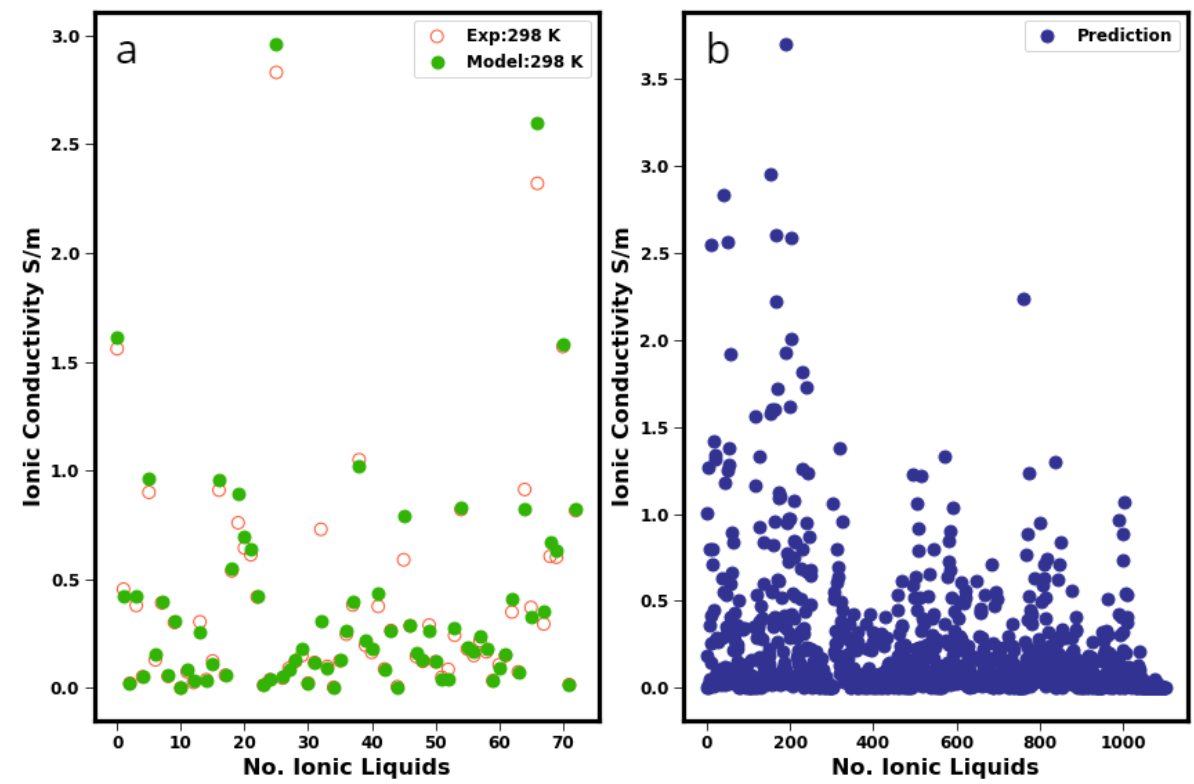

Figure 5: (a) Ionic conductivity comparison between experiment (open circle in red) and model prediction(green) using FFANN for all those data at $298 \mathrm{~K}$. (b) Unique ionic liquid predictions using FFANN for 1102 ionic liquid obtained by combining 29 unique cations and 38 anions at $298 \mathrm{~K}$. The ionic conductivity for these ionic liquids at 350 and $400 \mathrm{~K}$ appear in the supporting information (Figure $\mathbf{S 1 4}$ )

One potential issue with the generation of ionic liquids by combining cations and anions is the lack of knowledge concerning whether such ionic liquids would exist in the liquid state at the temperature of interest. For example, Martino et al. reported that the physical state of $\left[\mathrm{C}_{1} \mathrm{mim}\right][\mathrm{DCA}]$ is a supercooled liquid at room temperature. [70. In lieu of experiments, some clues into the physical state of these ionic liquids can be gleaned from conducting molecular simulations and analyzing the resulting radial distribution functions as performed by Beckner and Pfaendtner. 38. We decided not to purse such an approach as our primary motivation here is to identify pure ionic liquids, and binary ionic liquid mixtures bearing high ionic conductivity. In future studies, we plan to perform molecular simulations to offer insights into the molecular-level mechanism for high conductivity of the novel ionic liquids suggested by our model. We also hope that the promising ionic liquid candidates emerging from our work will enable the experimental community to focus its efforts in the discovery for high ionic conductivity ionic liquids.

\subsection{Binary Ionic Liquid Mixtures}

In this section, we evaluate the performance of the FFANN model in predicting ionic conductivity of binary ionic liquid mixtures using transfer learning, 
where the idea is to solve new tasks by transferring knowledge gained from a closely related problem. In this work, the transfer learning takes the form of using pure-component ionic conductivity data to develop a model to predict the ionic conductivity data for binary ionic liquids which the model has not encountered before. The utility of the approach stems from the fact that there is a significant increase in the number of binary ionic liquids due not only to the combinatorics but also the fact that the concentration of the constituent ionic liquids is now an additional independent variable. For example, if the number of unique cations is $N_{\mathrm{c}}$ and $N_{\mathrm{a}}$ is the number of anions, there are potentially $N_{\mathrm{c}} *\left(N_{\mathrm{c}}-1\right) / 2 * N_{\mathrm{a}}$ binary ionic liquids with common anion (Binary_C systems) and $N_{\mathrm{c}} * N_{\mathrm{a}} *\left(N_{\mathrm{a}}-1\right) / 2$ binary ionic liquids sharing the identical cation (Binary_A systems); the number of ionic liquids is further amplified by the number of practically realizable formulations. With 29 unique cations and 38 anions, we enumerated 15,428 Binary_C and 20,387 Binary_A systems. For each of these mixtures, we probed 19 intermediate concentrations spaced at an interval of 0.05 mole fraction between the pure ionic liquids leading to a total of $\sim 680,000$ binary ionic liquids.

For estimating the ionic conductivity of a given binary mixture, we combined the input features of the constituent ionic liquid cations and anions on a mole fraction basis. For example, for a Binary_C system designated as $[\mathrm{C} 1]_{x}[\mathrm{C} 2]_{1-x}[\mathrm{~A}]$, we obtained the cation features as the mole fraction-weighted average of the features for [C1] and [C2]. As this is an illustration for a common anion, we retained the input features for the anion as derived in the model development. Analogously, for Binary_A systems represented as $[\mathrm{C}][\mathrm{A} 1]_{x}[\mathrm{~A} 2]_{1-x}$, we kept the cation features while the anion features were derived by scaling the individual anion features by respective mole fractions and adding the features thus calculated. To examine the overall accuracy of such an approach, we compared the model predictions with experimental data reported for several binary ionic liquid mixtures in the NIST database and literature. 71, 72, 73, 74, 75, 76.

\subsection{Comparison of Experimental and FFANN-predicted Ionic Conductivity of Binary Ionic Liquids}

Table 2 lists the thirteen binary ionic liquid mixtures for which experimental data for ionic conductivity are available along with the number of data points and temperature range. Also included in Table 2 are the FFANN predictions for these systems and corresponding RMSD values. It is remarkable that the RMSD it is less than $0.1 \mathrm{~S} / \mathrm{m}$ for many systems, implying the suitability of the model for estimating the ionic conductivity for binary ionic liquid systems. 
Table 2: Root mean-squared deviation of the prediction and experimental data for binary ionic liquid mixtures. N.D stands for number of datapoints present in the dataset. $\left[\mathrm{C}_{4} \mathrm{mim}\right]^{*}$ stands for 1-butyl-2,3-dimethylimidazolium cation.

\begin{tabular}{|c|c|c|c|c|}
\hline System & N.D & $\begin{array}{r}\text { Temperature } \\
\text { Range/K } \\
\end{array}$ & $\begin{array}{r}\text { RMSD } \\
\mathrm{S} / \mathrm{m} \\
\end{array}$ & Reference \\
\hline$\left[\mathrm{C}_{2} \operatorname{mim}\right][\mathrm{DCA}]+\left[\mathrm{C}_{2} \mathrm{mim}\right]\left[\mathrm{BF}_{4}\right]$ & 9 & 298.15 & 0.46 & 772 \\
\hline$\left[\mathrm{C}_{2} \operatorname{mim}\right][\mathrm{DCA}]+\left[\mathrm{C}_{2} \mathrm{mim}\right][\mathrm{SCN}]$ & 30 & 298.15-323.15 & 0.36 & 73 \\
\hline$\left[\mathrm{C}_{4} \mathrm{mim}\right][\mathrm{Cl}]+\left[\mathrm{C}_{4} \operatorname{mim}\right]\left[\mathrm{CF}_{3} \mathrm{SO}_{3}\right]$ & 5 & 298.0 & 0.05 & 766 \\
\hline$\left[\mathrm{C}_{4} \mathrm{mim}\right]\left[\mathrm{MeSO}_{4}\right]+\left[\mathrm{C}_{4} \mathrm{mim}\right]\left[\mathrm{Me}_{2} \mathrm{PO}_{4}\right]$ & 4 & 298.0 & 0.17 & [76 \\
\hline$\left[\mathrm{C}_{4} \mathrm{mim}\right]\left[\mathrm{NTf}_{2}\right]+\left[\mathrm{C}_{4} \mathrm{mim}\right]\left[\mathrm{CF}_{3} \mathrm{SO}_{3}\right]$ & 5 & 298.0 & 0.05 & [76] \\
\hline$\left[\mathrm{C}_{4} \operatorname{mim}\right]\left[\mathrm{NTf}_{2}\right]+\left[\mathrm{C}_{4} \mathrm{mim}\right]\left[\mathrm{MeSO}_{4}\right]$ & 5 & 298.0 & 0.10 & 76 \\
\hline$\left[\mathrm{C}_{4} \operatorname{mim}\right]\left[\mathrm{NTf}_{2}\right]+\left[\mathrm{C}_{4} \mathrm{mim}\right]\left[\mathrm{Me}_{2} \mathrm{PO}_{4}\right]$ & 6 & 298.0 & 0.21 & [76] \\
\hline$\left[\mathrm{C}_{4} \mathrm{mim}\right]^{*}[$ Azide $]+\left[\mathrm{C}_{4} \mathrm{mim}\right]^{*}\left[\mathrm{BF}_{4}\right]$ & 70 & $303.15-368.15$ & 0.08 & [71] \\
\hline$\left[\mathrm{C}_{8} \operatorname{mim}\right][\mathrm{Cl}]+\left[\mathrm{C}_{8} \mathrm{mim}\right]\left[\mathrm{BF}_{4}\right]$ & 42 & 303.0-333.0 & 0.02 & [75] \\
\hline$\left[\mathrm{C}_{6} \operatorname{mim}\right][\mathrm{Cl}]+\left[\mathrm{C}_{6} \operatorname{mim}\right]\left[\mathrm{BF}_{4}\right]$ & 42 & $303.0-333.0$ & 0.06 & 755] \\
\hline$\left[\mathrm{C}_{4} \mathrm{mim}\right]\left[\mathrm{NTf}_{2}\right]+\left[\mathrm{C}_{4} \mathrm{mim}\right][$ Acetate $]$ & 32 & 283.15-333.15 & 0.13 & [74] \\
\hline$\left[\mathrm{C}_{6} \operatorname{mim}\right][\mathrm{Cl}]+\left[\mathrm{C}_{6} \operatorname{mim}\right]\left[\mathrm{PF}_{6}\right]$ & 35 & 303.0-333.0 & 0.07 & 75 \\
\hline$\left[\mathrm{C}_{2} \operatorname{mim}\right]\left[\mathrm{BF}_{4}\right]+\left[\mathrm{C}_{8} \operatorname{mim}\right]\left[\mathrm{BF}_{4}\right]$ & 30 & $280.0-300.0^{\dagger}$ & 0.09 & \\
\hline Overall & 315 & & 0.167 & \\
\hline
\end{tabular}

${ }^{\dagger}$ Personal communication
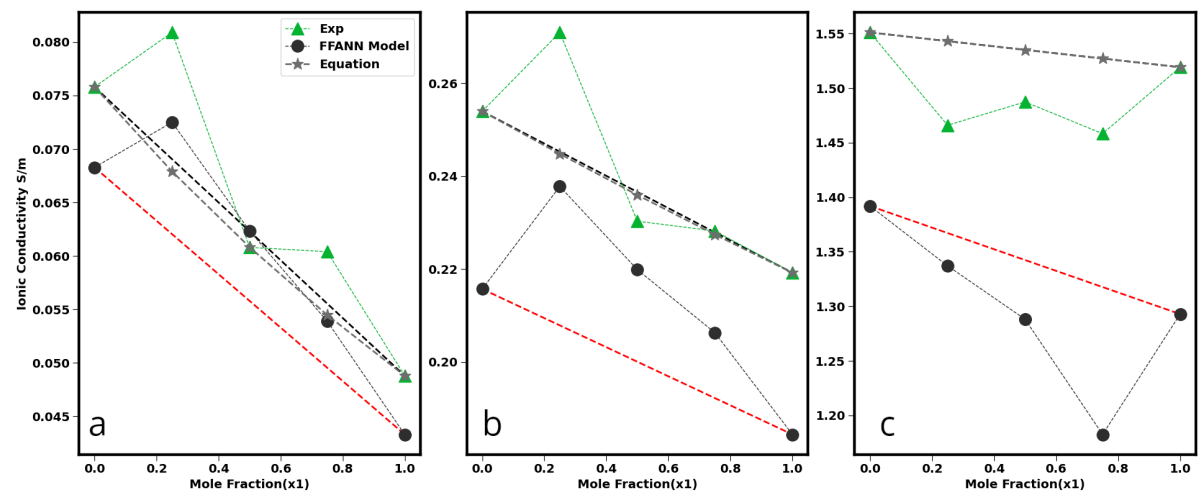

Figure 6: Comparison between experiment, FFANN model and log based linear relationship shown in equation ?? for $\left[\mathrm{C}_{4} \mathrm{mim}\right]^{*}[\text { Azide }]_{x 1}+\left[\mathrm{C}_{4} \mathrm{mim}\right]^{*}\left[\mathrm{BF}_{4}\right]_{1-x 1}$ at (a) $303.15 \mathrm{~K}$, (b) $323.15 \mathrm{~K}$ and (c) $368.15 \mathrm{~K}$. The dashed lines connecting the pure end points are only guide to the eye. $\left[\mathrm{C}_{4} \mathrm{mim}\right]^{*}$ stands for 1-butyl-2,3-dimethylimidazolium cation. 71] The dashed line with $\star$ is obtained by a logarithmic combining rule for ionic conductivity $\ln \sigma_{m i x}=$ $x_{1} * \ln \sigma_{1}+\left(1-x_{1}\right) * \ln \sigma_{2}$, while the dashed line without symbol indicates estimates with a linear combining rule.

Out of the thirteen systems examined in Table 2 the binary system comprised of $\left[\mathrm{C}_{4} \mathrm{mim}\right]^{*}[$ Azide $]$ and $\left[\mathrm{C}_{4} \mathrm{mim}\right]^{*}\left[\mathrm{BF}_{4}\right]$ exhibits non-ideal behavior, where the ionic conductivity achieves either a minimum or maximum at an intermediate 
mole fraction. Figure 6 shows that the ionic liquid passes through a maximum at lower temperatures ( 303 and $323 \mathrm{~K}$ ), while a minimum is observed at higher temperature $(368 \mathrm{~K})$. It is remarkable that the FFAAN model developed here is able to capture the trend despite its complexity. Furthermore, the model is accurate enough to identify the concentrations at which such extrema were measured in the experiment. [71] Overall this indicates that the model is robust enough to closely match both qualitative and quantitative trends; this is quite encouraging given that the data for these binary ionic liquid systems were not part of the model development. We further tested the predictive capability of the model to reproduce such a non-ideal behavior reported by McFarlane et al. [77. The authors measured the molar conductivity for the binary ionic system of $\left[\mathrm{C}_{2} \mathrm{mim}\right]\left[\mathrm{NTf}_{2}\right]$ and $\left[\mathrm{C}_{2} \mathrm{mim}\right]\left[\mathrm{CF}_{3} \mathrm{SO}_{3}\right]$ and found that a maximum at an intermediate mole fraction. Due to the lack of experimental data for molar volumes, a direct comparison was not possible; however, our model outputs (Figure S15) indeed confirmed that the binary ionic liquid mixture would exhibit a maximum in ionic conductivity.

Encouraged by the success of the model in estimating ionic conductivity for several binary mixtures, we proceeded to examine if there are binary ionic liquid mixtures producing an extremum (either a maximum or minimum) in ionic conductivity as the mole fractions of the constituent ionic liquids are varied. We discovered that there were a total of 5040 Binary_C systems, which yielded a maximum in the ionic conductivity. On the other hand, a total of 3771 Binary_A systems produced a maximum in the ionic conductivity at $298 \mathrm{~K}$. Normalizing these systems by the corresponding number of possible binary ionic liquid systems, we calculated that approximately $32.6 \%$ of Binary_C and $18.4 \%$ of Binary_A systems could potentially be formed to obtain ionic conductivity higher than those of the two pure ionic liquids forming the mixture. Two observations are worth pointing out: (a) binary ionic liquid systems offer a viable pathway for increasing ionic conductivity; (b) the likelihood for obtaining a maximum in ionic conductivity is higher when two different cations are mixed, particularly mixing cations with a large difference in the alkyl chain length.

In order to gain additional insight into the extent of enhancement in ionic conductivity, we calculated the percentage enhancement $(E)$ using eq. 1 where $\sigma_{\max }$ represents the maximum ionic conductivity for the mixture and $\sigma_{\text {max }}$,pure refers to the higher of the two pure ionic conductivities. Figure 7(a) and (b) present the binary enhancement factor for Binary_A and Binary_C systems, respectively.

$$
E=\frac{\sigma_{\max }-\sigma_{\text {max }, \text { pure }}}{\sigma_{\text {max }, \text { pure }}} * 100
$$

It is apparent that the percentage enhancement is large for the ionic liquid mixtures systems with ionic conductivity values lower than $1 \mathrm{~S} / \mathrm{m}$ and is partly attributable to the low conductivity values of the pure ionic liquids appearing in the denominator of eq. 1. It is also interesting to observe that the Binary_C systems display a broader range of enhancement values in comparison to those 
found for Binary_A systems. The analysis suggests that there exists at least one ionic liquid mixture for each of the unique cations and anions exhibiting an enhancement. We also uncovered that the Binary_A mixtures for which the maximum enhancement are the top three highest system containing hydrogen sulfate $\left[\mathrm{HSO}_{4}\right]^{-}$and chloride as the anions. These mixtures are (i) $\left[\mathrm{C}_{6} \operatorname{mim}\right][\mathrm{Cl}]_{0.75}\left[\mathrm{HSO}_{4}\right]_{0.25}$ with a maximum pure value of $0.0021 \mathrm{~S} / \mathrm{m}$ and enhanced maximum value of $0.0177 \mathrm{~S} / \mathrm{m}$ leading to an enhancement of $715.4 \%$, (ii) $\left[\mathrm{C}_{8} \mathrm{mim}\right][\mathrm{Cl}]_{0.75}\left[\mathrm{HSO}_{4}\right]_{0.25}$ with a maximum pure value of $0.0010 \mathrm{~S} / \mathrm{m}$ and enhanced maximum value of $0.008 \mathrm{~S} / \mathrm{m}$ with an enhancement of $675.4 \%$ and (iii) $\left[\mathrm{C}_{3} \mathrm{mim}\right][\mathrm{Cl}]_{0.55}\left[\mathrm{HSO}_{4}\right]_{0.45}$ with a maximum pure value of $0.006 \mathrm{~S} / \mathrm{m}$ and enhanced maximum value of $0.047 \mathrm{~S} / \mathrm{m}$ with an enhancement of $582.4 \%$. As for the binary cation mixture seen in (b), 1-(1-cyanomethyl)-3-methylimidazolium 0.6 3-(2-(butylamino)-2-oxoethyl)-1-ethyl-1H-imidazolium $0.4\left[\mathrm{PF}_{6}\right]$ has a maximum pure value of $0.0196 \mathrm{~S} / \mathrm{m}$ and the maximum value of $0.113 \mathrm{~S} / \mathrm{m}$ leading to an increase of $485.2 \%$.
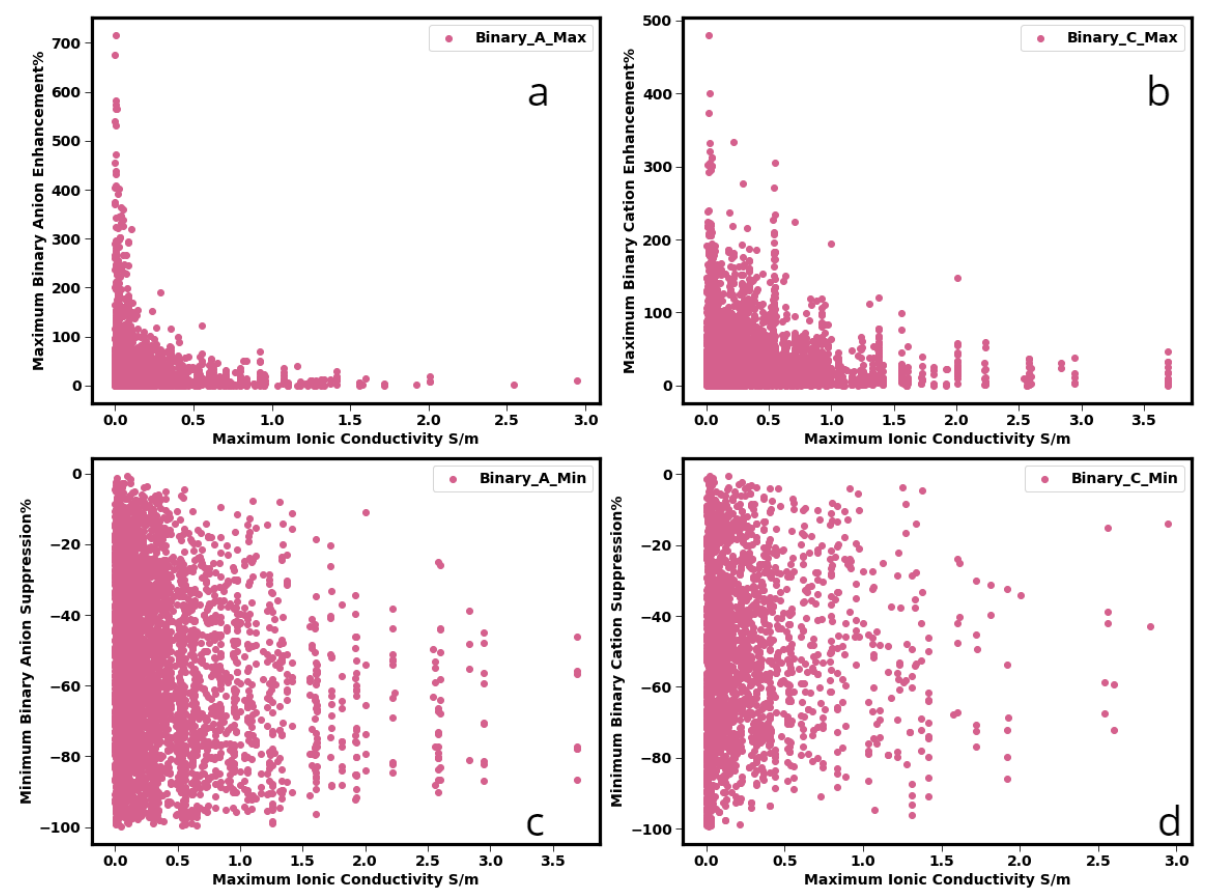

Figure 7: Percentage enhancement and suppression in ionic conductivity for binary ionic liquid mixtures at $298 \mathrm{~K}$. Binary_A_Max stands for a binary mixture sharing a common cation showing maximum enhancement; Binary_C_Max stands for a binary cation mixture displaying maximum enhancement; Binary_A_Min denotes a binary anion mixture exhibiting minimum suppression, and Binary_C_Min represents a binary cation mixture producing minimum suppression.

Similarly, there were several binary ionic liquid systems which showed an opposite behavior, i.e., there is at least one binary ionic liquid composition at which 
the ionic conductivity is lower than those of the corresponding pure ionic liquids. We uncovered 2305 Binary_C and 4284 Binary_A systems which showed a minimum in the ionic conductivity as a function of the ionic liquid composition at $298 \mathrm{~K}$. To quantify the extent of lowering in the ionic conductivity, we calculated percentage suppression $(S)$ using eq. 2 in which $\sigma_{\min }$ denotes the minimum in ionic conductivity and $\sigma_{\text {max,pure }}$ refers to the maximum of the two pure ionic conductivities. We elected to measure the deviation from $\sigma_{\max \text {,pure }}$ to emphasize the reduction in the

$$
S=\frac{\sigma_{\min }-\sigma_{\max , \text { pure }}}{\sigma_{\max , \text { pure }}} * 100
$$

ionic conductivity expected when an ionic liquid with lower conductivity is mixed with the one possessing high conductivity. Inherent in the definition in eq. 2 is the fact that the percentage lowering is capped at $100 \%$. The extent of depression in the ionic conductivity depicted in Figure 7 confirms the expectation. It is noteworthy that the suppression in the ionic conductivity brought about by the mixture of anions is restricted to ionic liquids with ionic conductivity below $1 \mathrm{~S} / \mathrm{m}$, while the depression in the ionic conductivity due to mixing of cations is predicted to cover the entire range of ionic conductivities. Furthermore, we identified the number for a given cation pair or anion responsible for elevating or depressing ionic conductivity for binary mixtures. The analysis is presented in the form of various heat maps (Figures S16, S17, S18, and $\mathrm{S19}$. It is also interesting to note that the short chain alkyl cations such as 1-methylimidazolium and 1,3-dimethylimidazolium are promising cations for ionic conductivity enhancement when combined with other cations as seen in Figure S18 


\section{Conclusion}

In this article, we made use of the NIST ILThermo Database to derive an artificial neural network model (FFAAN) and a support vector machine regression model for predicting ionic conductivity of pure imidazolium-based ionic liquids. The ionic conductivity values ranged over six orders of magnitude and covered temperatures from $275 \mathrm{~K}$ to $475 \mathrm{~K}$. The input features for the models were obtained using RDKit. The overall accuracy was found to be nearly identical for both the models. An examination of the predictions for the high ionic conductivity ionic liquids suggested superior performance for FFAAN, which was then employed for subsequent predictions.

Using 29 unique cations and 38 unique anions in the database, the ionic conductivity for all the possible combinations (1102 in total) were predicted at $298 \mathrm{~K}$. The procedure led to the identification of the ionic liquid $\left[\mathrm{C}_{1} \mathrm{mim}\right][\mathrm{DCA}]$ with the highest conductivity of $3.70 \mathrm{~S} / \mathrm{m}-30 \%$ higher than the highest ionic conductivity reported in the NIST ILThermo Database at $298 \mathrm{~K}$. The prediction was confirmed with the experimental data available in the literature. A simple procedure for combining the features on a mole fraction-weighted basis was devised to evaluate the predictive capability of the model for ionic liquid-ionic liquid mixtures. The results obtained with the approach showed that model was able to accurately capture the ionic conductivity for several binary for which experimental data exist.

The present study suggests a large number of binary mixture with non-ideal behavior in terms of the ionic conductivity. We encourage the experimental and molecular simulation communities to test the predictions. Confirmation of such non-ideality will increase the confidence in such models, while any deviations of the measured or computed properties from the predictions will enable a further refinement of the model. In either case, it is expected that the concerted effort between the experimental, molecular simulation, and machine learning approaches will accelerate materials discovery in the ionic liquids domain. 


\section{Acknowledgement}

The study is supported by the National Science Foundation (NSF) Award Number CBET-1706798. The authors would also like to thank Prof. Joshua Sangoro for providing the experimental conductivity data for the ionic liquid mixtures of $\left[\mathrm{C}_{2} \mathrm{mim}\right]\left[\mathrm{BF}_{4}\right]$ and $\left[\mathrm{C}_{8} \mathrm{mim}\right]\left[\mathrm{BF}_{4}\right]$.

\section{Supporting Information}

The supporting information contains cation and anion features used in the model development, IUPAC names and structures of the ions, temperature distribution profile for the experimental data points, experimental ionic conductivity distribution profile, heat map correlation of cation and anion features with ionic conductivity, statistics for tuning the number of hidden layers and the learning rate, comparison of the experimental and model-predicted ten highest and ten lowest ionic conductivities, variation of the ionic conductivity with alkyl chain length, dependence of ionic liquid conductivities on temperature,experiment and predicted data for alkylborate and alkylsulfate, examples of comparison between experimental and predictions from the model for binary ionic liquid mixtures.

\section{References}

[1] P. Wasserscheid, T. Welton, Ionic liquids in synthesis, John Wiley \& Sons, 2008.

[2] A. Pereiro, J. Araújo, J. Esperança, I. Marrucho, L. Rebelo, Ionic liquids in separations of azeotropic systems-a review, The Journal of Chemical Thermodynamics 46 (2012) 2-28.

[3] A. S. Paluch, P. Dhakal, Thermodynamic assessment of the suitability of the limiting selectivity to screen ionic liquid entrainers for homogeneous extractive distillation processes, ChemEngineering 2 (4) (2018) 54.

[4] P. Dhakal, J. A. Ouimet, S. N. Roese, A. S. Paluch, Mosced parameters for 1-n-alkyl-3-methylimidazolium-based ionic liquids: Application to limiting activity coefficients and intuitive entrainer selection for extractive distillation processes, Journal of Molecular Liquids 293 (2019) 111552.

[5] P. Dhakal, A. R. Weise, M. C. Fritsch, C. M. O'Dell, A. S. Paluch, Expanding the solubility parameter method mosced to pyridinium-, quinolinium, pyrrolidinium-, piperidinium-, bicyclic-, morpholinium-, ammonium-, phosphonium-, and sulfonium-based ionic liquids, ACS omega 5 (8) (2020) 3863-3877. 
[6] J. García, S. García, J. S. Torrecilla, F. Rodríguez, N-butylpyridinium bis(trifluoromethylsulfonyl) imide ionic liquids as solvents for the liquid-liquid extraction of aromatics from their mixtures with alkanes: Isomeric effect of the cation, Fluid phase equilibria 301 (1) (2011) 62-66.

[7] A. R. Hansmeier, M. Jongmans, G. W. Meindersma, A. B. de Haan, Lle data for the ionic liquid 3-methyl-n-butyl pyridinium dicyanamide with several aromatic and aliphatic hydrocarbons, The Journal of Chemical Thermodynamics 42 (4) (2010) 484-490.

[8] I. Domínguez, E. J. Gonzalez, Á. Domínguez, Liquid extraction of aromatic/cyclic aliphatic hydrocarbon mixtures using ionic liquids as solvent: literature review and new experimental lle data, Fuel processing technology 125 (2014) 207-216.

[9] A. Heintz, J. K. Lehmann, C. Wertz, J. Jacquemin, Thermodynamic properties of mixtures containing ionic liquids. 4. lle of binary mixtures of [c2mim][ntf2] with propan-1-ol, butan-1-ol, and pentan-1-ol and [c4mim][ntf2] with cyclohexanol and 1, 2-hexanediol including studies of the influence of small amounts of water, Journal of Chemical \& Engineering Data 50 (3) (2005) 956-960.

[10] D. R. MacFarlane, M. Forsyth, P. C. Howlett, J. M. Pringle, J. Sun, G. Annat, W. Neil, E. I. Izgorodina, Ionic liquids in electrochemical devices and processes: managing interfacial electrochemistry, Accounts of chemical research 40 (11) (2007) 1165-1173.

[11] M. Galiński, A. Lewandowski, I. Stepniak, Ionic liquids as electrolytes, Electrochimica acta 51 (26) (2006) 5567-5580.

[12] A. Lewandowski, A. Świderska-Mocek, Ionic liquids as electrolytes for li-ion batteries - an overview of electrochemical studies, Journal of Power Sources 194 (2) (2009) 601-609.

[13] A. A. Lee, D. Vella, S. Perkin, A. Goriely, Are room-temperature ionic liquids dilute electrolytes?, The journal of physical chemistry letters 6 (1) (2015) 159-163.

[14] X. Zhang, X. Zhang, H. Dong, Z. Zhao, S. Zhang, Y. Huang, Carbon capture with ionic liquids: overview and progress, Energy \& Environmental Science 5 (5) (2012) 6668-6681.

[15] A. Finotello, J. E. Bara, D. Camper, R. D. Noble, Room-temperature ionic liquids: temperature dependence of gas solubility selectivity, Industrial \& Engineering Chemistry Research 47 (10) (2008) 3453-3459.

[16] C. Cadena, J. L. Anthony, J. K. Shah, T. I. Morrow, J. F. Brennecke, E. J. Maginn, Why is co2 so soluble in imidazolium-based ionic liquids?, Journal of the American Chemical Society 126 (16) (2004) 5300-5308. 
[17] J. Jacquemin, P. Husson, V. Majer, M. F. C. Gomes, Influence of the cation on the solubility of co 2 and h 2 in ionic liquids based on the bis (trifluoromethylsulfonyl) imide anion, Journal of solution chemistry 36 (8) (2007) 967-979.

[18] H. Sakaebe, H. Matsumoto, N-methyl-n-propylpiperidinium bis (trifluoromethanesulfonyl) imide (pp13-tfsi)-novel electrolyte base for li battery, Electrochemistry Communications 5 (7) (2003) 594-598.

[19] S. García, M. Larriba, J. García, J. S. Torrecilla, F. Rodríguez, Liquidliquid extraction of toluene from n-heptane using binary mixtures of n-butylpyridinium tetrafluoroborate and n-butylpyridinium bis (trifluoromethylsulfonyl) imide ionic liquids, Chemical engineering journal 180 (2012) 210-215.

[20] H. Niedermeyer, J. P. Hallett, I. J. Villar-Garcia, P. A. Hunt, T. Welton, Mixtures of ionic liquids, Chemical Society Reviews 41 (23) (2012) 77807802 .

[21] P. Dhakal, J. K. Shah, Recent advances in molecular simulations of ionic liquid-ionic liquid mixtures, Current Opinion in Green and Sustainable Chemistry (2019).

[22] N. V. Plechkova, K. R. Seddon, Applications of ionic liquids in the chemical industry, Chemical Society Reviews 37 (1) (2008) 123-150.

[23] K. Paduszyński, Extensive Databases and Group Contribution QSPRs of Ionic Liquids Properties. 1. Density, Industrial \& Engineering Chemistry Research 58 (13) (2019) 5322-5338.

[24] W. Beckner, C. M. Mao, J. Pfaendtner, Statistical models are able to predict ionic liquid viscosity across a wide range of chemical functionalities and experimental conditions, Molecular Systems Design \& Engineering 3 (1) (2018) 253-263.

[25] K. Paduszyński, Extensive Databases and Group Contribution QSPRs of Ionic Liquids Properties. 2. Viscosity, Industrial \& Engineering Chemistry Research 58 (36) (2019) 17049-17066.

[26] V. Venkatraman, S. Evjen, H. K. Knuutila, A. Fiksdahl, B. K. Alsberg, Predicting ionic liquid melting points using machine learning, Journal of Molecular Liquids 264 (2018) 318-326.

[27] L. Cao, P. Zhu, Y. Zhao, J. Zhao, Using machine learning and quantum chemistry descriptors to predict the toxicity of ionic liquids, Journal of hazardous materials 352 (2018) 17-26.

[28] V. Venkatraman, B. K. Alsberg, Predicting co2 capture of ionic liquids using machine learning, Journal of CO2 Utilization 21 (2017) 162-168. 
[29] M. Mesbah, S. Shahsavari, E. Soroush, N. Rahaei, M. Rezakazemi, Accurate prediction of miscibility of $\mathrm{co} 2$ and supercritical $\mathrm{co} 2$ in ionic liquids using machine learning, Journal of CO2 Utilization 25 (2018) 99-107.

[30] H. Mokarizadeh, S. Atashrouz, H. Mirshekar, A. Hemmati-Sarapardeh, A. M. Pour, Comparison of LSSVM model results with artificial neural network model for determination of the solubility of $\mathrm{SO} 2$ in ionic liquids, Journal of Molecular Liquids 304 (C) (2020) 112771.

[31] K. Paduszyński, 2021, Extensive Databases and Group Contribution QSPRs of Ionic Liquid Properties. 3: Surface Tension, Ind. Eng. Chem. Res.

[32] D. Yalcin, T. C. Le, C. J. Drummond, T. L. Greaves, Machine Learning Approaches for Further Developing the Understanding of the Property Trends Observed in Protic Ionic Liquid Containing Solvents., The Journal of Physical Chemistry B 123 (18) (2019) 4085-4097.

[33] M. Hashemkhani, R. Soleimani, H. Fazeli, M. Lee, A. Bahadori, M. Tavalaeian, Prediction of the binary surface tension of mixtures containing ionic liquids using Support Vector Machine algorithms, Journal of Molecular Liquids 211 (C) (2015) 534-552.

[34] M. Hosseinzadeh, A. Hemmati-Sarapardeh, Toward a predictive model for estimating viscosity of ternary mixtures containing ionic liquids, Journal of Molecular Liquids 200 (PB) (2014) 340-348.

[35] J. S. Torrecilla, M. Deetlefs, K. R. Seddon, F. Rodríguez, Estimation of ternary liquid-liquid equilibria for arene/alkane/ionic liquid mixtures using neural networks, Physical Chemistry Chemical Physics 10 (33) (2008) 51147.

[36] T. L. Greaves, K. S. Schaffarczyk McHale, R. F. Burkart-Radke, J. B. Harper, T. C. Le, Machine learning approaches to understand and predict rate constants for organic processes in mixtures containing ionic liquids., Physical Chemistry Chemical Physics 23 (4) (2021) 2742-2752.

[37] F. Yusuf, T. Olayiwola, C. Afagwu, Application of Artificial Intelligencebased predictive methods in Ionic liquid studies: A review, Fluid Phase Equilib. 531 (2021) 112898.

[38] W. Beckner, J. Pfaendtner, Fantastic Liquids and Where To Find Them: Optimizations of Discrete Chemical Space, Journal of Chemical Information and Modeling 59 (6) (2019) 2617-2625.

[39] W. Beichel, Y. Yu, G. Dlubek, R. Krause-Rehberg, J. Pionteck, D. Pfefferkorn, S. Bulut, D. Bejan, C. Friedrich, I. Krossing, Free volume in ionic liquids: a connection of experimentally accessible observables from pals and pvt experiments with the molecular structure from xrd data, Physical Chemistry Chemical Physics 15 (22) (2013) 8821-8830. 
[40] P. Johansson, L. E. Fast, A. Matic, G. B. Appetecchi, S. Passerini, The conductivity of pyrrolidinium and sulfonylimide-based ionic liquids: a combined experimental and computational study, Journal of Power Sources 195 (7) (2010) 2074-2076.

[41] W. Beichel, U. P. Preiss, S. P. Verevkin, T. Koslowski, I. Krossing, Empirical description and prediction of ionic liquids' properties with augmented volume-based thermodynamics, Journal of Molecular Liquids 192 (2014) $3-8$.

[42] F. Gharagheizi, P. Ilani-Kashkouli, M. Sattari, A. H. Mohammadi, D. Ramjugernath, D. Richon, Development of a lssvm-gc model for estimating the electrical conductivity of ionic liquids, Chemical Engineering Research and Design 92 (1) (2014) 66-79.

[43] K. Tochigi, H. Yamamoto, Estimation of ionic conductivity and viscosity of ionic liquids using a qspr model, The Journal of Physical Chemistry C 111 (43) (2007) 15989-15994.

[44] R. L. Gardas, J. A. Coutinho, Group contribution methods for the prediction of thermophysical and transport properties of ionic liquids, AIChE Journal 55 (5) (2009) 1274-1290.

[45] Y. Chen, Y. Cai, K. Thomsen, G. M. Kontogeorgis, J. M. Woodley, A group contribution-based prediction method for the electrical conductivity of ionic liquids, Fluid Phase Equilibria (2020) 112462.

[46] Q. Dong, C. D. Muzny, A. Kazakov, V. Diky, J. W. Magee, J. A. Widegren, R. D. Chirico, K. N. Marsh, M. Frenkel, Ilthermo: a free-access web database for thermodynamic properties of ionic liquids, Journal of Chemical \& Engineering Data 52 (4) (2007) 1151-1159.

[47] Q. Dong, A. F. Kazakov, C. D. Muzny, R. D. Chirico, J. A. Widegren, V. Diky, J. W. Magee, K. N. Marsh, M. D. Frenkel, Ionic liquids database (ilthermo), Tech. rep. (2006).

[48] https://pypi.org/project/pyilt2/, accessed: 2020-09-03.

[49] M. Kanakubo, K. R. Harris, N. Tsuchihashi, K. Ibuki, M. Ueno, Temperature and pressure dependence of the electrical conductivity of 1-butyl-3methylimidazolium bis (trifluoromethanesulfonyl) amide, Journal of Chemical \& Engineering Data 60 (5) (2015) 1495-1503.

[50] J. Vila, B. Fernández-Castro, E. Rilo, J. Carrete, M. Domínguez-Pérez, J. Rodríguez, M. García, L. Varela, O. Cabeza, Liquid-solid-liquid phase transition hysteresis loops in the ionic conductivity of ten imidazoliumbased ionic liquids, Fluid Phase Equilibria 320 (2012) 1-10. 
[51] M. Vranes, S. Dozic, V. Djeric, S. Gadzuric, Physicochemical characterization of 1-butyl-3-methylimidazolium and 1-butyl-1-methylpyrrolidinium bis (trifluoromethylsulfonyl) imide, Journal of Chemical \& Engineering Data 57 (4) (2012) 1072-1077.

[52] S. Dožić, N. Zec, A. Tot, S. Papović, K. Pavlović, S. Gadžurić, M. Vraneš, Does the variation of the alkyl chain length on $\mathrm{n} 1$ and $\mathrm{n} 3$ of imidazole ring affect physicochemical features of ionic liquids in the same way?, The Journal of Chemical Thermodynamics 93 (2016) 52-59.

[53] K. R. Harris, M. Kanakubo, N. Tsuchihashi, K. Ibuki, M. Ueno, Effect of pressure on the transport properties of ionic liquids: 1-alkyl-3methylimidazolium salts, The Journal of Physical Chemistry B 112 (32) (2008) 9830-9840.

[54] B. Mbondo Tsamba, S. Sarraute, M. Traïkia, P. Husson, Transport properties and ionic association in pure imidazolium-based ionic liquids as a function of temperature, Journal of Chemical \& Engineering Data 59 (6) (2014) 1747-1754.

[55] T. Makino, M. Kanakubo, Y. Masuda, H. Mukaiyama, Physical and co 2-absorption properties of imidazolium ionic liquids with tetracyanoborate and bis (trifluoromethanesulfonyl) amide anions, Journal of Solution Chemistry 43 (9-10) (2014) 1601-1613.

[56] Y.-H. Yu, A. N. Soriano, M.-H. Li, Heat capacities and electrical conductivities of 1-n-butyl-3-methylimidazolium-based ionic liquids, Thermochimica acta 482 (1-2) (2009) 42-48.

[57] K. Fukumoto, M. Yoshizawa, H. Ohno, Room temperature ionic liquids from 20 natural amino acids, Journal of the American Chemical Society 127 (8) (2005) 2398-2399.

[58] J.-G. Li, Y.-F. Hu, C.-W. Jin, H.-D. Chu, X.-M. Peng, Y.-G. Liang, Study on the conductivities of pure and aqueous bromide-based ionic liquids at different temperatures, Journal of solution chemistry 39 (12) (2010) 18771887.

[59] E. I. Izgorodina, R. Maganti, V. Armel, P. M. Dean, J. M. Pringle, K. R. Seddon, D. R. MacFarlane, Understanding the effect of the c 2 proton in promoting low viscosities and high conductivities in imidazolium-based ionic liquids: part i. weakly coordinating anions, The Journal of Physical Chemistry B 115 (49) (2011) 14688-14697.

[60] D. M. Lowe, P. T. Corbett, P. Murray-Rust, R. C. Glen, Chemical name to structure: Opsin, an open source solution (2011).

[61] https://opsin.ch.cam.ac.uk/, accessed: 2020-03-28.

[62] http://www.rdkit.org/, accessed: 2020-03-28. 
[63] F. Pedregosa, G. Varoquaux, A. Gramfort, V. Michel, B. Thirion, O. Grisel, M. Blondel, P. Prettenhofer, R. Weiss, V. Dubourg, J. Vanderplas, A. Passos, D. Cournapeau, M. Brucher, M. Perrot, E. Duchesnay, Scikit-learn: Machine learning in Python, Journal of Machine Learning Research 12 (2011) 2825-2830.

[64] V. Vapnik, The nature of statistical learning theory, Springer science \& business media, 2013.

[65] M. Hashemkhani, R. Soleimani, H. Fazeli, M. Lee, A. Bahadori, M. Tavalaeian, Prediction of the binary surface tension of mixtures containing ionic liquids using support vector machine algorithms, Journal of Molecular Liquids 211 (2015) 534-552.

[66] C. Zhao, H. Zhang, X. Zhang, M. Liu, Z. Hu, B. Fan, Application of support vector machine (svm) for prediction toxic activity of different data sets, Toxicology 217 (2-3) (2006) 105-119.

[67] K. Matsumoto, E. Nishiwaki, T. Hosokawa, S. Tawa, T. Nohira, R. Hagiwara, Thermal, physical, and electrochemical properties of li [n (so2f) 2][1-ethyl-3-methylimidazolium][n (so2f) 2] ionic liquid electrolytes for li secondary batteries operated at room and intermediate temperatures, The Journal of Physical Chemistry C 121 (17) (2017) 9209-9219.

[68] G. Saito, Y. Yoshida, Development of conductive organic molecular assemblies: organic metals, superconductors, and exotic functional materials, Bulletin of the Chemical Society of Japan 80 (1) (2007) 1-137.

[69] Y. Yoshida, O. Baba, G. Saito, Ionic liquids based on dicyanamide anion: influence of structural variations in cationic structures on ionic conductivity, The Journal of Physical Chemistry B 111 (18) (2007) 4742-4749.

[70] W. Martino, J. F. De La Mora, Y. Yoshida, G. Saito, J. Wilkes, Surface tension measurements of highly conducting ionic liquids, Green Chemistry 8 (4) (2006) 390-397.

[71] Y. O. Andriyko, W. Reischl, G. E. Nauer, Trialkyl-substituted imidazoliumbased ionic liquids for electrochemical applications: basic physicochemical properties, Journal of Chemical \& Engineering Data 54 (3) (2009) 855-860.

[72] A. Stoppa, R. Buchner, G. Hefter, How ideal are binary mixtures of roomtemperature ionic liquids?, Journal of Molecular Liquids 153 (1) (2010) $46-51$.

[73] P. D. Bastos, F. S. Oliveira, L. P. Rebelo, A. B. Pereiro, I. M. Marrucho, Separation of azeotropic mixtures using high ionicity ionic liquids based on 1-ethyl-3-methylimidazolium thiocyanate, Fluid Phase Equilibria 389 (2015) 48-54. 
[74] M. Kanakubo, T. Makino, T. Umecky, Co2 solubility in and physical properties for ionic liquid mixtures of 1-butyl-3-methylimidazolium acetate and 1-butyl-3-methylimidazolium bis (trifluoromethanesulfonyl) amide, Journal of Molecular Liquids 217 (2016) 112-119.

[75] H. Ning, M. Hou, Q. Mei, Y. Liu, D. Yang, B. Han, The physicochemical properties of some imidazolium-based ionic liquids and their binary mixtures, Science China Chemistry 55 (8) (2012) 1509-1518.

[76] M. T. Clough, C. R. Crick, J. Gräsvik, P. A. Hunt, H. Niedermeyer, T. Welton, O. P. Whitaker, A physicochemical investigation of ionic liquid mixtures, Chemical science 6 (2) (2015) 1101-1114.

[77] H. Every, A. Bishop, M. Forsyth, D. R. Macfarlane, Ion diffusion in molten salt mixtures, Electrochimica Acta 45 (8-9) (2000) 1279-1284. 